\title{
Oro-facial Injuries and Mouth Guard use in British National League Water Polo Players
}

\author{
Joel Thomas1*, Robert Grant Jagger ${ }^{2}$ and Andrew Curran ${ }^{3}$ \\ ${ }^{1}$ Oral and Maxillofacial Department, University Hospitals Bristol, UK \\ ${ }^{2}$ Clinical Lecturer, University of Bristol, Bristol, UK \\ ${ }^{3}$ Dentist, Horfield Dental Care, Horfield, Bristol, UK
}

Received: April 17, 2015; Accepted: May 20, 2015; Published: June 15, 2015

*Corresponding author: Joel Thomas, Oral and Maxillofacial Department, University Hospitals Bristol, UK Bristol Dental School, Lower Maudlin Street, Bristol, BS12LY, E-mail: jt6215@my.bristol.ac.uk

\begin{abstract}
Objectives: The objective of this cross-sectional study is to assess the prevalence of facial, oral and dental injuries and frequency of mouth guard use amongst British Men's National Division 1 water polo players.

Material and methods: All National League Division 1 water polo club squads were included in the study. Participants were asked to complete a questionnaire that sought information regarding injuries and mouth guard use.

Results: One hundred and sixteen completed questionnaires were returned ( $87 \%$ response rate). Ninety four percent of subjects reported having experienced at least one facial, oral or dental injury whilst playing water polo. Of the injuries sustained, those to the lips and tongue were the most prevalent (84.5\%). Facial injuries were also common. Forty percent of those injured, reported having sustained at least one dental injury, of which tooth fracture was the most common. More than seventy percent believed that mouth guards were effective at preventing injury but mouth guards were worn regularly when playing by only 8 respondents.
\end{abstract}

Conclusions: It was concluded that facial, oral and dental injuries were very common. Seventy percent believed that mouth guards were effective at preventing injury but mouth guards were worn regularly during matches by few respondents.

Keywords: Dental trauma; Facial injury; Water polo

\section{Introduction}

Water polo has been considered to be a medium risk sport for dental trauma [1]. It is not considered to be a contact sport, though limited physical contact against the ball carrier is allowed within the rules. Holding and wrestling a player to maintain or gain position to receive, pass or shoot a ball is an important part of the game. Injuries in water polo however are common. The shoulders, hands and face are the regions of the body most frequently injured [2-4].

The shoulder may be affected because of repeated, explosive movements of the shoulder and arm during passing and shooting actions. Tearing or straining of the small rotator-cuff muscles responsible for stabilising the shoulder joint is a common problem. Injuries to the elbow, wrist and finger joints can be sustained by a defensive player when trying to block a shot by putting an arm up to defend the goal. The impact of the ball upon the arm or hand can cause sprain, dislocation or fracture.

Trauma to the face and mouth can be caused by a ball thrown at speed. It can also occur as a result of players making contact whilst swimming in close proximity using a front crawl stroke and also when wrestling for position or ball possession or when a player's arm, following through after releasing a shot, strikes the defensive player who guards them. These events can traumatise the bony prominences of the face such as the nose, zygomatic arches and the teeth, and cause associated soft tissue trauma such as contusion and laceration. Other head injuries include supra-orbital lacerations, facial bone fracture, tympanic membrane perforation and eye trauma such as blow out fractures and corneal abrasions. Whilst it has been determined that injury is commonly sustained to the face and mouth [4] there is little information regarding the details of those injuries and in particular regarding the dental injuries sustained.

There is compelling evidence regarding the protective capacity of sports mouth guards [5,6]. The use of mouth guards in water polo however is not mandatory and little is known about prevalence of mouth guard use amongst water polo players.

The aim of the study was to determine the prevalence of facial, oral and dental injuries among male water polo players competing in the British National League Division 1 Water Polo league. The prevalence of mouth guard use among those players was also determined.

\section{Materials and Methods}

All players in all ten teams forming the British Men's Water Polo National League Division 12012 were included in the study (Table 1). The list of current teams within the British National League can be found at www.bwpl.org. Team managers of each team were contacted to seek permission to include their team 
Table 1: Questionnaire response rate from all Division 1 teams.

\begin{tabular}{|c|c|c|}
\hline Team & Squad members & $\begin{array}{c}\text { Questionnaire } \\
\text { Responses }\end{array}$ \\
\hline Cheltenham & 15 & 15 \\
\hline Lancaster & 15 & 12 \\
\hline Bristol & 15 & 10 \\
\hline Manchester & 15 & 12 \\
\hline Portobello & 15 & 14 \\
\hline Rotherham & 15 & 9 \\
\hline London Penguins & 15 & 12 \\
\hline Invicta & 15 & 11 \\
\hline London Polytechnic & 15 & 10 \\
\hline Welsh Wanderers & 15 & 11 \\
\hline Total & & 116 \\
\hline
\end{tabular}

members in the study. All agreed to participate. Information sheets and questionnaires were given to each team manager to distribute to their squad members. The information sheet described the purpose of the study and invited participants to complete the questionnaire. The questionnaire sought information about facial, oral and dental injuries sustained while playing water polo and about the subjects' use of protective sports mouth guards (Table 2). Ethical approval for the study was obtained from the University of Bristol, Faculty of Medicine and Dentistry Committee for Research Ethics.

Questionnaires were completed anonymously by subjects and were placed into envelopes by respondents and returned to the managers. Team managers returned all envelopes containing completed questionnaires to the investigators.

Data were transferred to a personal computer using Microsoft® Office Excel 2003.

Table 2: The questionnaire.

1. Do you wear a mouth guard when playing:

$$
\text { Always Mostly Occasionally Never }
$$

2. Do you think mouthguards are effective in protecting teeth and the mouth?

$$
\text { Yes No Don't know }
$$

3. If you DO wear a mouth guard, where did you get it?

$$
\text { Dentist Sports shop Somewhere else (please specify) }
$$

4. What types of injuries have you sustained during playing? Please indicate the number of times it has occurred.

\begin{tabular}{|l|l|l|}
\hline Area & Number of times & \\
\hline Teeth & & Please also complete question 5 \\
\hline Face & & Please also complete question 6 \\
\hline Lips and tongue & & Please also complete question 7 \\
\hline
\end{tabular}

5. Teeth

\begin{tabular}{|l|l|l|}
\hline Type of injury & Number of times & Total teeth \\
\hline Broken tooth & & \\
\hline Loosened tooth & & \\
\hline Knocked out tooth & & \\
\hline
\end{tabular}

6. Face Injury

\begin{tabular}{|l|l|}
\hline Type of injury & Number of times \\
\hline Cut & \\
\hline Fracture/Broken Bone & \\
\hline
\end{tabular}

7. Lip and tongue

\begin{tabular}{|l|l|}
\hline Type of injury & Number of times \\
\hline Cut lip & \\
\hline Cut tongue & \\
\hline Bruised/swollen lip & \\
\hline
\end{tabular}




\section{Results}

All teams $(\mathrm{n}=10)$ provided responses. One hundred and sixteen players completed the questionnaire $(87 \%$ response rate). Mean number of responses per team was 11.6 (range 9 - 15). Mean age of respondents was 26.1 years (range 15 - 51). Non-response was due to players opting not to complete the questionnaire.

\section{Prevalence of Injuries}

One hundred and nine players (94\% of all respondents) reported at least one injury. The total number of injuries reported was 1297 . The mean number of reported injuries per player was 11.2 .

\section{Distribution of Injuries}

The number of respondents sustaining the different types of injury is shown in Table 3. Lip and tongue injuries (85\%) were most common and dental injuries (41\%) least common.

Facial injuries: Eighty five (73\%) respondents had sustained an injury to the face. A total of 238 facial cuts and 28 facial fractures were reported. Thirteen $(11 \%)$ respondents had fractured a facial bone. Five (4\%) of respondents reported having fractured a facial bone on more than one occasion.

Lip and tongue injuries: These were the most commonly reported injury, being reported by 98 players with a mean of 9.5 such injuries per player.

Dental injuries: Forty eightsubjects (41\%) reported suffering damage to a tooth. The number of respondents sustaining the different types of injury is shown in Table 4 . Fractured teeth were the most commonly reported dental injury (40 subjects, 34\%). Loosened and avulsed teeth were less common (6 (5\%) and 7 (6\%) respectively).

Some subjects reported suffering more than one type of dental injury and a total of 96 dental injuries were reported. Table 5 shows the number of dental injuries per player. The number of injuries per player ranged from 0 to 6 , with a mean of 2.0 injuries per player. Six point nine percent of subjects had sustained 4 or more dental injuries.

\section{Mouth guard use}

In response to the question 'Do you think that mouth guards

Table 3: Number of players reporting facial injuries.

\begin{tabular}{|c|c|c|}
\hline & $\mathbf{n}$ & $\mathbf{( \% )}$ \\
\hline Dental Injury & 48 & 41 \\
\hline Facial & 85 & 73 \\
\hline Lip and Tongue & 98 & 85 \\
\hline
\end{tabular}

Table 4: Number of players reporting dental injuries.

\begin{tabular}{|c|c|c|}
\hline Dental Injuries: & Yes (\%) & No (\%) \\
\hline Any dental injury & $48(41)$ & $68(59)$ \\
\hline Fractured tooth & $40(34)$ & $76(66)$ \\
\hline Loosened tooth & $6(5)$ & $110(95)$ \\
\hline Avulsed tooth & $7(6)$ & $109(94)$ \\
\hline
\end{tabular}

Table 5: Frequency of dental injuries.

\begin{tabular}{|c|c|c|c|c|c|c|}
\hline \multirow{2}{*}{$\begin{array}{c}\text { Not } \\
\text { injured } \\
(n(\%))\end{array}$} & \multirow{2}{*}{$\begin{array}{l}\geq 1 \text { in- } \\
\text { jury } \\
(n(\%))\end{array}$} & \multicolumn{4}{|c|}{$\begin{array}{l}\text { Number of dental injuries } \\
\text { experienced (n (\%)) }\end{array}$} & \multirow{2}{*}{$\begin{array}{c}\text { Total } \\
\text { subjects }\end{array}$} \\
\hline & & 1 & 2 & 3 & $\geq 4$ & \\
\hline 68 & $\begin{array}{c}48 \\
(41.4)\end{array}$ & $21(18.1)$ & $\begin{array}{c}13 \\
(11.2)\end{array}$ & $\begin{array}{c}6 \\
(5.2)\end{array}$ & $8(6.9)$ & 116 \\
\hline
\end{tabular}

Table 6: Comparison of number of players (\%) reporting injuries in UK and Swiss water polo players.

\begin{tabular}{|c|c|c|c|c|}
\hline \% & $\begin{array}{c}\text { Any dental } \\
\text { injury }\end{array}$ & $\begin{array}{c}\text { Loosened } \\
\text { tooth }\end{array}$ & $\begin{array}{c}\text { Displaced } \\
\text { Tooth }\end{array}$ & $\begin{array}{c}\text { Fractured } \\
\text { tooth }\end{array}$ \\
\hline UK & 41 & 5 & 6 & 34 \\
\hline Switzerland & 21 & 1.4 & 3.4 & 16.4 \\
\hline
\end{tabular}

are effective in protecting teeth and the mouth?', eighty two $(70.6 \%)$ believed that mouth guards were effective, eight players (7\%) believed that they were not effective and twenty six (22.4\%) were uncertain of their benefit.

Twelve subjects $(10 \%)$ reported having worn a mouth guard at sometime. Four players always wore a mouth guard when playing, four players mostly and four players occasionally wore a mouth guard. One hundred and four players (90\%) reported never having worn a mouth guard.

Ten of the subjects had obtained their mouth guard from a dentist. Two had bought mouth guards from a sports shop.

\section{Discussion}

This investigation has demonstrated that injuries to the face, lips and teeth are commonly sustained by British National League first division water polo players when playing water polo. More than $90 \%$ of respondents had suffered oro-facial trauma at some time whilst playing. More than $40 \%$ reported having sustained damage to a tooth whilst playing.

The questionnaire used in this study was similar to those used in previous studies [7,8]. As discussed previously the findings of such questionnaires must be interpreted with some caution [8] because the retrospective design means that data are subjective and accuracy is reliant on the memory of the subjects. It is possible that minor injuries were forgotten or ignored for reporting purposes. Those subjects who had experienced multiple traumatic events may have only estimated the number of events. Further, the types of injury may not be mutually exclusive. For example, damage to the lips may coincide with damage to the teeth. An individual might list both these relating to one blow or may only state the most important one. The figures may be an underestimate since players may have suffered knocks and bruises, which they considered to be insignificant or did not remember. The reported frequency of soft tissue injuries in particular is of more doubtful accuracy. Significant events such as bone or tooth fractures and tooth avulsions should have been reported with some accuracy.

The results of this study confirm the findings of previous studies that oral and facial injuries are highly prevalent in water polo [2-4]. The majority of injuries were to the lips and tongue, although a significant percentage occurred to other facial regions. 
The most common injury to other facial regions was laceration of the supra-orbital region and this is in agreement with the finding of Franic et al [4]. Although most facial injuries were soft tissue trauma or bruising, many had suffered dental trauma and fractures of facial bones. These hard tissue injuries may be considered the most severe injuries sustained as these require significant medical intervention including long term follow up. Most players had suffered multiple injuries.

There has previously been little information about the prevalence and nature of the different types of dental injuries sustained by water polo players. A recent study reported dental injuries in 415 Swiss water polo players [9]. In that study the participating players came from Swiss national leagues A and $\mathrm{B}$, first and second leagues, as well as the women's and junior's league. A comparison of the findings with the present study is shown in Table 6 . The distribution of the types of dental injury was similar in both groups. The number of fractured teeth in both studies was high. The prevalence of dental injuries was much greater in the present study. This might reflect the high level of competitiveness in the British National League. In addition, the Swiss study examined the frequency of dental and facial injuries in water polo in players of a wider ability.

The distribution and prevalence of dental injuries differs from that previously reported for other sports. In hockey, for example, avulsed teeth are relatively more common than fractured teeth [7]. The difference could be explained by the damage in hockey being caused by stick or ball. It is also possible that the relatively high number of loosened teeth could be due to a mouth guard dissipating the traumatic force resulting in loosening of the teeth rather than fracture. In the hockey study, $88 \%$ of respondents regularly wore a mouth guard.

It was not possible to determine accurately from the questionnaires whether or not a mouth guard was worn when dental damage occurred when playing water polo however only eight of the respondents in the present study wore a mouth guard regularly.

It is perhaps surprising that so few respondents wore a mouth guard because $70 \%$ believed mouth guards to be effective. Mouth guards restrict breathing and make communication difficult. Water polo is played at high intensity, so any effect on breathing is a major consideration. However there is strong evidence for effectiveness [5,6]and participants in other sports now have widespread acceptance of the use of mouth guards.

Oro-facial and dental injuries are highly prevalent amongst top level water polo players. It is therefore important for coaches, trainers and swimming pool staff to be able to implement appropriate management measures and be able to facilitate access to further medical or dental care if needed.

The present study has identified that first division water polo players have a high risk of oro-facial injuries. It is recognised that risk of injury can increase with the level at which a sport is played, both because of increased commitment and increased time spent playing and training. The Swiss study showed that dental damage occurred at all levels of the sport. Further studies at all levels of the sport are needed to determine more precisely risk of injury and arrangements in place for emergency medical and dental care. It is clear that there is also a need for the promotion of mouth guard wearing amongst water polo players.

\section{Conclusions}

It is concluded that facial, oral and dental injuries were very common. Seventy percent of respondents believed that mouth guards were effective at preventing injury yet mouth guards were worn regularly during matches by very few players.

\section{What Are The New Findings?}

1. $94 \%$ of British division one water polo players reported experiencing at least one oro-facial injury whilst playing.

2. $85 \%$ of players had experienced lip and tongue injuries, $41 \%$ had experienced dental injuries and $11 \%$ had experienced fracture of the facial bones during play.

3. $90 \%$ of players had never worn a mouth guard whilst playing.

4. $70 \%$ of players believed mouth guards to be effective.

\section{How Might It Impact On Clinical Practice In The Near Future?}

1. Water polo coaches should be made aware of appropriate management of oro-facial injuries.

2. Dental professionals should strongly advise the use of mouth guards when playing water polo.

3. Further investigation is indicated with regard to why mouth guards are not worn by water polo players.

\section{References}

1. Federation Dentaire International. Guidelines for dental protection during sporting activities; Drugs and sport.1990; FDI, London, UK.

2. Brooks JM. Injuries in water polo. Clin Sports Med 1999; 18(2):313-9.

3. Annett $P$, Fricker $P$, McDonald W. Injuries to elite water polo players over a 13 year period. NZ Sports Med J. 2000; 28:78-83.

4. Franic M, Ivkovic A, Rudic R. Injuries in water polo. Croat Med J. 2007; 48(3):281-8.

5. Marshall SW, Loomis DP, Waller AE et al. Evaluation of protective equipment for prevention of injuries in rugby union. Int J Epidemiol. 2005; 34(1):113-8.

6. Knapik JJ, Marshall SW, Lee RB, Darakjy SS, Jones SB, Mitchener TA, et al. Mouthguards in sport activities : history, physical properties and injury prevention effectiveness. Sports Med. 2007; 37(2):117-44.

7. Hendrick K, Farrelly P, Jagger R. Oro-facial injuries and mouth guard use in elite female field hockey players. Dent Traumatol 2008; 24(2):189-92. doi: 10.1111/j.1600-9657.2007.00527.x.

8. Jagger RG, Abbasbhai A, Patel D, Jagger DC, Griffiths A. The prevalence of dental, facial and head injuries sustained by school boy rugby players. A pilot study. Prim Dent Care. 2010; 17(3):143-6. doi: 10.1308/135576110791654793.

9. Hersberger S, Krastl G, Kuhl S, Filippi A. Dental injuries in water polo, a survey of players in Switzerland. Dent Traumatol. 2012; 28(4):28790. doi: 10.1111/j.1600-9657.2011.01083.x. 